\title{
Análisis desde la educación universitaria de la asociatividad en los sectores pesqueros artesanales: caso Chanduy, Santa Rosa, y La Libertad
}

Analysis from the university education of the associativity in the artisanal fishing sectors: Chanduy, Santa Rosa, and

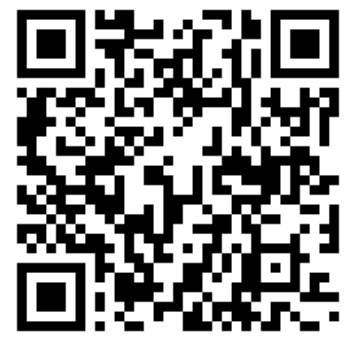
La Libertad case

Hermelinda Cochea Tomalá**

Humberto Peña Rivas ${ }^{* * *}$

Isabel Balón Ramos ${ }^{* * * *}$

Margarita Panchana Panchana ${ }^{* * * * *}$

\section{Resumen}

Los cambios estructurales en materia política en el Ecuador en los últimos 10 años, han provocado que las condiciones en la que opera la economía se encuentren en modificación constante, esto ha

\footnotetext{
* Artículo original derivado del Proyecto "Educación universitaria en asociaciones" Financiado por la Universidad Estatal Península de Santa Elena entre junio 2018 y mayo 2019

** Docente Universitario. Universidad Estatal Península De Santa Elena. Guayaquil, Ecuador. E-mail: hermelindacochea@hotmail.com. ORCID: http://orcid.org/0000-0002-6625-1166

*** Docente Universitario. Universidad Estatal Península De Santa Elena. Guayaquil, Ecuador. E-mail: hpenarivas@hotmail.com ORCID: http://orcid.org/0000-0002-4528-7936

**** Docente Universitario. Universidad Estatal Península De Santa Elena. Guayaquil, Ecuador. E-mail: isablebalon@gmail.com._ ORCID: http://orcid.org/0000-0003-2672-4683

***** Docente Universitario. Universidad Estatal Península De Santa Elena. Guayaquil, Ecuador. E-mail: margaritapanchana@gmail.com. ORCID: http://orcid.org/0000-0002-8392-2216
}



Atribución/Reconocimiento-NoComercialCompartirlgual 4.0 Licencia Pública Internacional - CC BY-NC-SA 4.0 https://creativecommons.org/licenses/by-ncsa/4.0/legalcode.es 
obligado a que, las Asociaciones Sociales replanteen un modelo productivo diferente a los esquemas tradicionales, para adaptarse a las nuevas tendencias de organización social. Frente a esta situación, el presente trabajo tiene como principal objetivo, Promover el desarrollo de una gestión moderna mediante un modelo de asociatividad para el sector pesquero artesanal de los puertos ubicados en la Comuna Chanduy, Santa Rosa y La Caleta en la Provincia de Santa Elena de la República del Ecuador. Unos de los factores que tiene mayor influencia en las Asociaciones es la globalización económica, lo obliga a las empresas a aplicar estrategias con el único fin de lograr una mayor eficiencia.. La metodología de investigación se caracteriza por utilizar un enfoque cuantitativo y cualitativo, mediante la aplicación de método deductivo y analítico, lo que permite medir las percepciones y expectativas de los involucrados para la recopilación de información específica para el desarrollo de nuestro objeto de estudio. Finalmente, este trabajo investigativo permitió llegar a los siguientes criterios como son; la escasez de asociaciones y su organización, y se pudo determinar que las estrategias del modelo son importantes para las asociaciones ya que esto permite asegurar la sostenibilidad de los recursos manteniendo activa la pesca artesanal de una manera eficiente que logre el crecimiento económico de este importante sector de la Región.

Palabras clave: asociatividad, modelos administrativos, eficiencia administrativa

\begin{abstract}
The structural changes in political matters in Ecuador in the last 10 years, have caused that the conditions in which the economy operates are in constant modification, this has forced the Social Associations to rethink a productive model different from traditional schemes, to adapt to new trends in social organization. Faced with this situation, the present work has as main objective, Promote the development of a modern management through a model of associativity for the artisanal fishing sector of the ports located in the Chanduy Commune, Santa Rosa and La Caleta in the Province of Santa Elena of the Republic of Ecuador. One of the factors that has the greatest influence in the Associations is economic globalization,
\end{abstract}


it forces companies to apply strategies with the sole purpose of achieving greater efficiency. The research methodology is characterized by using a quantitative and qualitative approach, through the application of deductive and analytical method, which allows to measure the perceptions and expectations of those involved for the collection of specific information for the development of our object of study. Finally, this research work allowed us to reach the following criteria as they are; the scarcity of associations and their organization, and it was possible to determine that the strategies of the model are important for the associations since this allows to ensure the sustainability of the resources keeping active the artisanal fishing in an efficient way that achieves the economic growth of this important sector

Key words: associativity, administrative models, administrative efficiency

\section{Introducción}

En los puertos artesanales de las parroquias de Chanduy, Santa Rosa y Caleta del Cantón La Libertad - Provincia de Santa Elena, la principal fuente de ingreso se basa en el desarrollo de la actividad pesquera artesanal, sus productos son llevados a los puertos para su comercialización. Actualmente el ciudadano dedicado a esta actividad mantiene desconocimientos sobre los beneficios que ofrece la asociatividad e implementación de estrategias administrativas para la gestión de sus recursos, es por ello que se encuentran trabajando de forma independiente lo que no permite aprovechar las ventajas competitivas que se generan bajo un esquema de asociatividad comercial y artesanal. Por otro lado, existe el incumplimiento de compromisos adquiridos por créditos otorgados y de los pagos mensuales y anuales que deben cumplir, estos hechos que han incidido para que las dos únicas asociaciones fueran suprimidas de las diferentes organizaciones del Ministerio de Agricultura, Ganadería, Acuacultura y Pesca (MAGAP), Ministerio de Industrias y Productividad (MIPRO), Ministerio de Inclusión Económica y Social (MIES).

Ante estas problemáticas, surge la necesidad de propuesta de un modelo de Asociatividad como estrategia para el fortalecimiento de 
la actividad pesquera, lo cual permitirá que los pescadores mejoren su gestión comercial. La presente investigación se basa en promover la asociatividad y potencializar capacidades en los pescadores menos favorecidos, aumentar el índice de producción dentro de estas parroquias a través de un Modelo Asociativo que contribuya a optimizar la producción pesquera.

Se fundamenta la aplicación del presente proyecto en el ámbito social, los pescadores obtendrán más conocimientos en los beneficios que puede traer el trabajo mediante la asociatividad con otros de su comunidad, generando en ellos una visión empresarial que podrán aprovechar para emprender nuevos retos que les permita mejorar su entorno actual. Por el lado del aspecto económico, se logrará que los pescadores tengan un ingreso más estable, que conjuntamente con la consolidación de la asociación se traducirá en la mejora de su economía y a su vez obtener una mejor calidad de vida.

Según (Morán). El Gobierno Nacional trabaja para fortalecer el sector pesquero artesanal Los modelos asociativos desarrollados en diferentes partes del país y en el exterior reflejan que las empresas que se sometieron a un modelo de asociatividad tuvieron un progreso significativo la cual les sirvió para expandirse ayudando así a estas personas a poder sobrevivir en el mercado. La metodología propuesta se basa en un enfoque descriptivo con medición de indicadores cuantitativos y cualitativos, y está encaminado al descubrimiento de los escasos modelos de asociatividad que existen en esta región, cabe recalcar que a través de este, se podrá definir las premisas que servirán como base para el desarrollo del modelo de asociatividad,

Con respecto a los niveles organizativos de las actividades económicas ya mencionadas, es necesario señalar que conforme al diagnóstico realizado, se confirma que son pocos los pescadores que se encuentran afiliados a una organización comunitaria, se puede evidenciar una problemática con respecto al nivel de organización de los pescadores, lo cual influye directamente en el ingreso económico, los cuales son insuficientes para una adecuada gestión comercial (Chanduy, 2018). 
Con respecto a los apartados que componen el presente proyecto, primeramente, se presenta una descripción del entorno en que se desarrollan los elementos que son objeto de estudio, sobre todo en las poblaciones mencionadas, y su relación con la actividad de pesca artesanal, se definen el objetivo y propuesta a desarrollar. En el literal II de describe el desarrollo del trabajo de investigación, se refiere a los modelos de asociatividad, sus componentes y la forma en que estos interactúan con grupos sociales, permitiendo implementar procesos de forma organizadas y hacia un objetivo en común. En el literal III de hace referencia a la metodología utilizada, para finalmente en el apartado IV se muestran los resultados obtenidos y los aspectos concluyentes de la investigación.

La asociatividad es un sistema de constantes cambios en la política actual, la misma que desarrolla mecanismos de acción conjunta y cooperación, siendo grupos asociativos que aspiran lograr tener un posicionamiento en el mercado con su contribución, lo cual les permitiría contar con una estructura más sólida y competitiva que les facilite enfrentarse a grandes retos para potenciar logros, contando con un respaldo económico sostenible.

La falta de un plan de desarrollo para una eficiente gestión de las actividades de pesca artesanal incide en el crecimiento socioeconómico de la región peninsular, en las poblaciones de Chanduy, La Libertad y Santa Rosa.

Por lo tanto, la asociatividad funciona como un mecanismo de cooperación, que proporciona a los pescadores artesanales la unión entre ellos, con el fin de resolver los problemas ligados a esa actividad de forma conjunta. Además, del mejoramiento del proceso productivo y una mejor posición en el mercado.

La elaboración del modelo de asociatividad está fundamentada en el artículo 66 numeral 15 de la (Banco Central del Ecuador, 2016), donde indica que cada ciudadano tiene "El derecho a desarrollar actividades económicas, en forma individual o colectiva, conforme a los principios de solidaridad, responsabilidad social y ambiental". Por otro lado, la Ley Orgánica de Economía Popular y Solidaria considera a las asociaciones como una organización económica que cumplen funciones de producción, comercialización, intercambio, 
financiación; por ende la puesta en práctica de un modelo de asociatividad sería un mapa que viabilice la ruta a seguir de los pescadores artesanales del puerto pesquero de Chanduy, Santa Rosa y La caleta.

De tal manera la implementación de esta estrategia va orientada al surgimiento de maximización de beneficios y de reducción de costos que permitan tener una mayor rentabilidad a sus actividades productivas, teniendo presente como responsabilidad social $\mathrm{y}$ ambiental el uso de la tecnología y que esta genere un sistema integral para la innovación, mejorando así mismo la organización dentro de las asociaciones o cooperativas. Promover el desarrollo de una gestión moderna mediante un modelo de asociatividad para el sector pesquero artesanal de los puertos ubicados en la Comuna Chanduy, Santa Rosa y La Caleta en la Provincia de Santa Elena.

Las fundamentaciones teóricas que sirven de base para el presente trabajo de investigación tienen sustento en la legislación ecuatoriana, sobre todo en materia de asociatividad y pesca artesanal, y en argumentaciones de autores que manifiestan la importancia de la organización social en los entornos socio-económicos de una determinada región.

El ser humano necesitó asociarse de diferentes formas desde la época primitiva, con la finalidad de conseguir sus objetivos y lograr la subsistencia, que en ese entonces eran la alimentación y la convivencia social (Biblioteca Univesidad Tecnologica de El Salvador, 2003).

Para (Salazar, 2007) Historiador de la Fundación Universitaria Luis Amigó de Colombia, en su estudio titulado asociatividad, redes sociales y desarrollo local, menciona que la asociatividad tiene sus orígenes en el ámbito filosófico, integracionista y contemporáneo.

En la parte filosófica Aristóteles, coincidía en que la asociatividad se origina por parte de la asociación humana, ya que sus causas y consecuencias permitían a estos establecer debates, llegando a un consenso presentando propuestas de organización de la sociedad, pensando siempre en el desarrollo y satisfacción de las personas. 
En lo referente al ámbito integracionista Husslein y Drucker, mencionaban que con el pasar del tiempo el conocimiento de las personas cambia, motivo por el cual el modelo integracionista $u$ organicista, fue tomando más importancia, ya no bastaba con la asociación como tal, sino que se necesitaban estructuras sociales dentro de un proceso asociativo, que permitieran alcanzar los objetivos de los grupos humanos. Y la asociatividad es contemporánea porque subsiste al mismo tiempo que otros factores en las empresas, de tal forma estudiarla es fundamental para ir descubriendo debilidades y carencias de la sociedad para desarrollar modalidades, con la finalidad de hacer frente a los cambios y retos que el mundo globalizado está presentando (Salazar, 2007).

Entonces se puede mencionar que la asociatividad tiene su origen desde el mismo momento en que el hombre creó esta necesidad, no obstante, con el transcurrir del tiempo y con el desarrollo del conocimiento y pensamiento del hombre, la asociatividad, sufrió cambios significativos, desarrollándose notablemente y creando diferentes modelos, los cuales se adaptaron a las necesidades, permitiendo un funcionamiento correcto $\mathrm{y}$ organizado de las personas que se asociaban con una finalidad común.

Asociatividad es una modalidad de cooperación que actualmente se está desarrollando en muchos países, teniendo gran acogida en el medio en el que se desempeña, debido a la efectividad e importancia que tiene para quienes la desarrollan, es por eso que antes de definir un concepto de lo que es asociatividad, se debe conocer los siguientes términos:

Según (Franco, 2009) manifiesta que: "Asociatividad es comprometerse por medio de la identificación de objetivos y metas habituales en organizaciones que se dedican a la misma o similar acción económica, dedicación total de manera individual, en metas de progreso de los diversos niveles del trabajo organizacional, que permita la sustentabilidad y ayude al crecimiento empresarial en los ambientes competitivos de los negocios, de esta manera facilitando las mejores condiciones para enfrentar la reñida competencia a nivel global. 
En la obra (Iguera, 2010) se menciona que: "La asociatividad puede adoptar diversas modalidades, dependiendo del objetivo por el cual se produce, cada modalidad implica diversas formas de participación de los actores y genera mayores o menores responsabilidades según sea el caso".

Cada modelo de asociatividad es diferente, debido a su objetivo; esto nos dice que cada empresa necesita su propia forma de asociatividad, ya que, cada organización tiene diferentes formas de realizar y afrontar problemas o actividades que se estén realizando en ellas para esto contamos con una clasificación donde lograremos identificar varias relaciones intraempresariales o varios conjuntos formados por estos.

Dentro de este proceso existen algunos elementos diferenciadores entre los términos distrito industrial, clúster y red empresarial, vale la pena destacar los elementos que tienen en común y que aportan a la construcción del proceso de asociatividad:

No excluyen a ninguna empresa por el tipo de mercado en el que opera.

Permiten la resolución de problemas conjuntos (HINESTRO, 2005).

Para poder desarrollar un modelo de asociatividad, es necesario seguir un proceso el cual sin duda alguna es muy importante, ya que, a través de este se logrará enrumbar el camino de manera segura y sobre todo consciente de lo que se quiere realizar.

Los modelos asociativos intervienen de forma positiva en los negocios, ya que contribuyen significativamente en su progreso. El porcentaje de asociatividad renueva en cada país según la calidad y soporte que se le esté ofreciendo por parte de las administraciones particulares o seccionales, gestión que debe ser fortalecida para que el contorno asociativo tenga los efectos esperados y consiga favorecer el aumento en los sectores productivos.

El MIPRO se basa a sus estudios revela que: las fortalezas de sus emprendedores son la juventud y la capacidad para descubrir 
oportunidades en el mercado. En cambio, las debilidades son la falta de capital y el poco aprendizaje del emprendedor.

Según el MIPRO, el emprendimiento a través de la asociatividad puede ser un motor de la economía ecuatoriana, pero su impacto aún es bajo, motivo por el cual solo es cuestión de seguir apoyando esta modalidad y a las personas que estén dispuestas a salir adelante a través de emprendimientos o modelos asociativos.

Según (MARCO HERRERA, 2015) La pesca artesanal en nuestro país es una actividad ancestral que evolucionó desde una pesca de subsistencia con artes y embarcaciones sencillas, hasta embarcaciones rápidas construidas de material de "fibra de vidrio" y barcos de madera. La tecnología es fundamental en esta labor brindando facilidades para las faenas de pescas; sin embargo a la actividad pesquera se la aprecia como un trabajo rentable siendo una ocupación muy reconocida a nivel mundial.

"Con un promedio de 18 mil embarcaciones en el país y 3 pescadores por cada una, según datos de la Federación Nacional de Cooperativas de Pesca del Ecuador (Fenacopec), la pesca artesanal genera un promedio de \$ 100 millones en exportaciones al año" (TELEGRAFO, 2108).

En el Ecuador la mayor parte de sus habitantes se dedican a la actividad pesquera, de tal manera que es considerando como un trabajo digno y productivo para el país, el mismo que necesita de mucho esfuerzo junto con dedicación constante por parte de las personas que la emplean; además la pesca es destinada para el consumo interno y para el comercio exterior generando grandes beneficios, resulta ser de vital importancia que contribuye con el desarrollo social y económico del Ecuador.

Los pescadores artesanales en la comuna Chanduy, enfrentan varios obstáculos para lograr su desarrollo como: conflictos, falta de interés de las personas, atrasos de cuotas de pagos en la asociatividad; pero a su vez podemos decir que la pesca es una actividad que representa una fuente de ingreso económico para las personas que se dedican a la explotación de este recurso, además se considera una fuente 
generadora de empleo que aporta a la producción interna bruta de los diferentes países que se dedican a esta actividad.

Según (Gabriela, 2008) indica que las pequeñas empresas son el motor del crecimiento de cualquier país. En el caso de Ecuador, representan alrededor de un 50 por ciento del PIB, y contribuyen con alrededor del 90\% de la tasa de empleo; El Ecuador es un país con una alta tasa de nacimiento de empresas, pero con la misma facilidad con la que se forman, la mayoría de estas empresas no logran superar los cinco años de vida.

Existe un mayor número de habitantes que se dedican a esta actividad, sin embargo el proceso de la captura de estas especies es complicado ya que realizan su faena de pesca para capturarlos.

La pesca artesanal de mar es significativa tanto en el consumo doméstico como en la industria peninsular. El comercio es la complementaria para comercializar los productos hacia las ciudades, como de éstas para cubrir las necesidades internas del cantón y la provincia.

Para (Baigún, 2013) la pesca artesanal involucra a múltiples actores $\mathrm{y}$ a todos los aspectos relacionados con el desenvolvimiento y el soporte de la pesca propiamente dicha, así como los procesos de postcaptura, procesamiento y mercadeo. Una de las características fundamentales de la pesca artesanal es su carácter sistémico porque integra varias dimensiones y conforman una red sostenida por componentes ecológicos, biofísicos, económicos, sociales y culturales interrelacionados a través de diferentes subsistemas.

La actividad pesquera debe ser concebida como un recurso que proporciona empleo y seguridad alimentaria a través del consumo directo de pescado y la posibilidad de adquirir bienes y gozar de servicios básicos como producto de los beneficios económicos que la pesca depara.

Para lo cual se deben desarrollar buenas prácticas pesqueras, que se garantice una pesca artesanal de modo sostenible en el tiempo en la parroquia Chanduy, para asegurar el bienestar económico y social de los sectores dependientes de esta actividad. 
De primera instancia las estrategias de crecimiento son una opción fundamental que las asociaciones, generalmente se plantean para una mejora del nivel competitivo o el fortalecimiento de la posición ya alcanzada.

Por ende, la alternativa adecuada es aquella que afronta las circunstancias que impiden el desarrollo comercial y productivo de un sector; por lo cual, tiene como propósito principal lograr un crecimiento en ventas de las diferentes asociaciones.

De la misma forma, se da a conocer la siguiente estrategia que pueden ayudar al crecimiento de las asociaciones basadas en las necesidades del mercado y la demanda del producto en los sectores pesqueros:

Básicamente el modelo tiene 2 fuentes de financiamiento:

1) Recursos propios de la asociación que se lo obtiene a través de las cuotas mensuales de socios y aportes extraordinarios de acuerdo a las necesidades de la institución.

2) Y la autogestión de recursos que pueden ser de dos tipos: prestación de servicios como el de facilitadores de las instituciones de gobierno o privadas para temas de capacitaciones y la de recursos económicos que se pueden obtener a través de la formulación de proyectos productivos que se realizarían con la asociación.

Dentro del sector pesquero uno de los temas principales de esta actividad es la comercialización, debido a que esta acción es la responsable de la demanda del producto, generando así beneficios a quienes la realizan, pero dentro de este mercado se observa que los principales beneficiarios son los intermediarios (canales de distribución), motivo por el cual se requiere plantear una estrategia para evitar estas interferencias dentro del sector pesquero. Se propone implementar una estrategia asociativa, con el que se busca mejorar la capacidad productiva de los pescadores artesanales, a través de la intervención de las instituciones gubernamentales y no gubernamentales, quienes serán los encargados de impulsar la asociación de los pescadores, motivando la integración y creación de estrategias que mejoren su rentabilidad. 
La comercialización asociativa radica en la conexión de varios emprendedores que buscan desarrollar una actividad sostenible, así mismo fortalecer la independencia laboral dentro de las comunidades pesqueras, también es un mecanismo de desarrollo que se da por la escasez de herramientas para negociar y comercializar, además poder reducir costos de distribución.

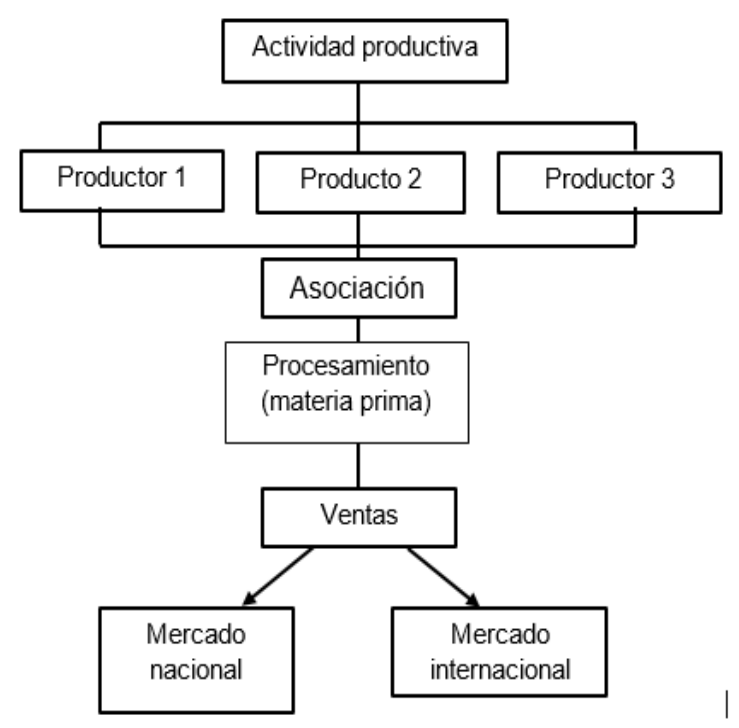

Figura 1 Estrategias de comercialización

Este tipo de asociatividad se la realiza para vender grandes volúmenes de producto pesquero, ingresando dentro del mercado competitivo mediante la negociación y la colocación de precios en cantidades altas, por ende, a través de esta estrategia se busca crear alternativas de manera positiva que permitan reducir los costos y comercialización del producto, mejorando el rendimiento $\mathrm{y}$ rentabilidad de esta actividad a largo plazo, además la oportunidad de obtener financiamiento para inversiones futuras e identificar nuevos mercados nacionales e internacionales para posicionar el producto pesquero con valor agregado y generar más ingresos mediante la participación en conjunto.

El marketing como estrategia aporta las herramientas necesarias para la aplicación de las mismas en una asociación de pescadores, el cual tiene como propósito impulsar y posicionar a la institución, de tal manera que sea productiva; así también trabajar por el desarrollo del sector pesquero artesanal de La Caleta, Santa Rosa y Chanduy. Por 
consiguiente, se enfoca en un sistema de alianzas estratégicas que le permiten cumplir con objetivos comerciales y aportar al mejoramiento de la calidad de vida de los pescadores artesanales.

Al realizar alianzas estratégicas, las asociaciones incrementan sus capacidades y destrezas, tanto en comercialización y captura, de tal manera que se ofrecerán productos de calidad a precios económicos, así se evitan los intermediarios entre el pescador y el consumidor, es decir los comerciantes no participan directamente con los pescadores en la negociación, por ende, los márgenes de rentabilidad son equitativos para los integrantes de la organización.

De esta manera, las asociaciones de pescadores artesanales se constituirán en líderes del mercado, proveedor de productos del mar al por mayor y por menor en los puertos de La caleta, Santa Rosa y Chanduy, es decir, en los mejores aliados estratégicos y con amplias ventajas competitivas por su nivel de organización, poder de negociación y capacidad de gestión

La asociatividad se entiende como una serie de acuerdos de participación o colaboración mutua, utilizada como estrategia para el beneficio de un negocio o emprendimiento (López , López, Pineda, \& Venegas, 2010). Considerando a (Risea, 2013) manifiesta que las instituciones para asociarse deben realizar un gran esfuerzo en establecer objetivos y políticas habituales. Es decir, las instituciones modifican estrategias donde puedan relacionarse entre sí, para lo cual de esa manera alcanzar los objetivos propuestos y enfrentarse al proceso de globalización.

La participación de la asociatividad se la considera una ventaja debido a que es el conjunto de esfuerzo que puede materializarse de diferentes maneras, a partir de la contratación del personal para la compra o venta de los productos o servicios pagados colectivamente, hasta la creación de una institución con naturaleza jurídica y capital propio con garantía para acceder al financiamiento de la comercialización de productos.

La asociatividad aparece como uno de los elementos fundamentales entre las asociaciones que se encuentran desafiado por el desarrollo económico nacional. Dando paso a nuevas oportunidades de trabajos 
para la sociedad pero esto también significa una amenaza para las asociaciones las cuales están presionadas a cambiar constantemente sus estrategias de funcionamiento, por ende requieren nuevos elementos de interacción con la sociedad para cumplir con sus objetivos.

Las estrategias factibles para que las asociaciones enfrenten la competitividad se origina desde los directivos de la institución, las cuales dependerán del tamaño y la cooperación de los individuos que conformen la institución, además pueden apelar a ciertas alternativas que se clasifican en dos categorías: los individuales y las colectivas que se encuentran relacionadas por un fin común. Las estrategias individuales se basan en la discrecionalidad y la eficiencia del representante del establecimiento, por otra parte las colectivas requiere de numerosos participantes de la misma para llevar a cabo los objetivos planteados.

Las estrategias individuales no se excluyen de las estrategias colectivas, como se mencionó anteriormente, ya que abarcan a todos los colaboradores de la institución y las acciones descienden de los diferentes objetivos, por ende el impacto generado dependerá de las características de las estrategias que se aplicarán.

La necesidad de innovar, diseñar y aumentar estrategias colectivas no solamente es para el progreso de la misma sino también para fortalecer las ventajas competitivas tanto individuales y colectivas, las cuales pueden llegar hacer un factor primordial para el desarrollo de las asociaciones o cooperativas. Además varias de las estrategias individuales pueden lograr el éxito a medida que el proceso va evolucionando lo cual tendrá mayor eficiencia al complementarse con estrategias colectivas.

La asociatividad proyecta puntos importantes, a conocer:

Son voluntarios.

Al ser de tipo colectivo, permiten generar diversas estrategias de desarrollo. 
Los conflictos se manejan de manera conjunta entre los diversos miembros de la asociación.

Estructura alianzas estratégicas para la extracción, comercialización y transporte de los productos del mar en óptimas condiciones para cubrir la demanda del mercado.

Para ser realmente efectiva, una ventaja competitiva debe ser:

Difícil de imitar.

Única.

Posible de mantener.

Ágil para otorgar un valor agregado.

Capaz de innovar y cambiar sus expectativas acorde a las situaciones.

Mantener una ventaja competitiva podría significar reforzar dicha ventaja hasta hacerla más duradera y significativa, es por aquello que la competitividad engloba muchos ámbitos que a su vez ayuda a convertir las ventajas en oportunidades.

Estas ventajas competitivas se pueden dar en diferentes aspectos de la asociación, por ejemplo puede haber una gran ventaja en el producto, precio, calidad, servicio, ubicación, infraestructura, entre otros.

Una asociación debe desarrollar los siguientes puntos para conseguir una ventaja competitiva:

Contar con una moderna infraestructura e implementación de nuevos equipos que le permita al producto estar en perfectas condiciones.

Mantener a un personal altamente calificado que garantice mayor productividad y excelente servicio al cliente.

Estar asentados en una ubicación estratégica para facilitar el acceso de los clientes. 
Al contar con nuevos mercados que ayude a la correcta distribución del producto la demanda y oferta será notoria en los mencionados sectores de pesca artesanal, teniendo una organización adecuada y cumpliendo con los reglamentos acordados para una distribución y desarrollo eficiente.

Característica de la asociatividad

Crecimiento poblacional y por ende su demanda.

Modernización, orden, genera valor agregado y la formalidad de realizar sus actos, sin inconvenientes regidos a las leyes

Tipos, cualidades, variedad de compradores y canales de distribución existentes.

Permite tener un nivel de debilidades decrecientes en conocimientos y capacidades.

Ser reconocidos a nivel nacional por otras asociaciones.

Obtener nuevas estrategias para la comercialización, abastecimiento, distribución y venta.

Capital relevante.

Conocimientos tecnológicos y profesionales.

Autoridad, poder, economía.

Ganancias de la asociatividad

Permite tener un nivel de debilidades decrecientes en conocimientos y capacidades, sea este trabajo realizado en equipo o individual.

Fortalecer el posicionamiento en el mercado dando el trato justo y equitativo para todos los socios.

Ubicación y horarios estratégicos para las respectivas actividades. 
Reducir el impacto de los costos que se dan para mantenimiento de embarcaciones.

Beneficios de la asociatividad

Control adecuado para los recursos administrativos y/o financieros.

Mayor productividad.

Conocimientos para una buena negociación, estrategias o alianzas.

La cooperación provoca el mejoramiento de la tecnología en sus procesos que faciliten el trabajo de las personas.

Existe solvencia en el caso de generarse riesgos incluso e incluso la compartición de costos.

La calidad de los productos o servicios es notable, generando incrementos en producción

La gestión técnica, productiva y comercial tiene que ser satisfactoria.

Dar realce al sector pesquero mejorando la infraestructura.

Mantiene estable la autonomía de los socios y se mejora el conocimiento técnico -productivo y comercial.

\section{GENERACIÓN DE LINEAMIENTOS}

Canal de distribución.

El canal de distribución es el medio por el cual un individuo entrega al consumidor final un determinado bien. Lo que se procura alcanzar con la creación de dicho grupo asociativo entre los pescadores artesanales es que se puedan eliminar los intermediarios y por medio de eso se logre entregar el producto de una manera directa, para que por medio de ello obtener más reconocimiento en el mercado y más acogida por parte del mismo. 


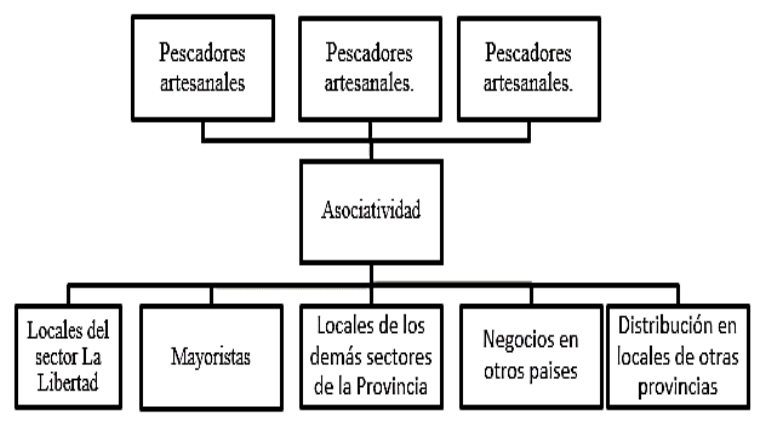

Figura 2 Canal de distribución

Desarrollo de mercados y procesos comerciales.

La estrategia de las asociaciones se encuentra en la implementación de un plan de desarrollo de mercado y procesos comerciales que pueden convertirse en uno de los canales de comercialización de los productos de forma eficiente y eficaz, con la posibilidad de que el producto a distribuir llegue a diferentes lugares de comercio, con una adecuada organización en los procesos empezando desde el pedido, la captura, envió del producto hasta el cobro final, por ende, esta estrategia permite una venta directa entre pescadores artesanales y consumidores sin la necesidad de intermediarios.

Además los intermediarios en la actualidad son los principales agentes de comercio debido a que establecen una conexión entre pescadores y consumidores al momento de llegar al puerto a desembarcar, es así que con esta estrategia se busca cortar esta cadena de fuerza para conseguir la sostenibilidad económica directamente del pescador.

También facilita el desarrollo de especialización en los pescadores de una asociación transformando pausadamente sus experiencias en conocimientos que sirven de ayuda en el desarrollo de mercado y procesos comerciales, esto permitiría reducir significativamente el precio del producto y de esta manera obtener una producción sustentable de manera diaria y una pesca responsable de calidad, permitiendo mejoras trascendentales desde la captura hasta la ventas de cada uno de este producto marino, garantizando así el cuidado de especies en tiempo de veda, por ende, para continuar con un proceso 
de comercialización adecuado se deberán seguir los siguientes lineamientos:

Uso de la tecnología: Páginas web, WhatsApp, Facebook.

Ponerse en contacto con empresas que necesiten del producto: Atuneras, Sardineras, Restaurantes.

Acordar un sitio físico para la entrega

Gestión sustentable de la actividad productiva.

Una estrategia de gestión sustentable de la actividad productiva esta direccionada a la sostenibilidad y asociatividad como ejes principales del desarrollo para alcanzar el éxito, esta debe estar enfocada con el cuidado medio ambiental para que tengan una conservación de los recursos naturales, haciendo uso adecuado de residuos sólidos para que no afecte a la actividades productivas de la pesca.

Por otra parte, la asociatividad promueve la creación de un fondo de ámbito financiero que requerirá la inversión de los socios, con el afán de otorgar préstamos internamente, y así minimizar la dependencia con las instituciones financieras públicas o privadas, porque estos son los que captan y acumulan la riqueza del sector y no permite el desarrollo.

La asociatividad es la relación con organismos públicos y privados que apoyan en la obtención de un fin común, a través de las ayudas políticas, sociales hasta entidades de financiamiento que fortalecen los múltiples proyectos y permite que los pescadores puedan estar incentivados para:

Tener el acceso de nuevas tecnología para una adecuada navegación de la mayoría de los pescadores asociados.

Adquirir un mejoramiento en las prácticas de manipulación e higiene adecuado del producto pesquero. 
Desarrollar más el arte de pesca en conjunto con métodos que les permita implementar un sistema de almacenamiento y conservación con su única finalidad de que incrementen la obtención de producto de primera y así mejorar la situación económica de los pescadores.

La ferretería y el abastecimiento de materiales de pesca.

La actividad pesquera lleva consigo una serie de técnicas al momento de ponerla en práctica y encontrarse realizando sus faenas en el mar es un poco arriesgado, es por ello que con el apoyo de todos los socios (pescadores) se podrá lograr tener un pequeño almacén de compra y venta de materiales o herramientas de pesca y poder generar esa comodidad en los puertos La caleta, Santa Rosa y Chanduy, además que dichos puertos pesqueros obtengan en un solo lugar todo lo necesario para poder realizar la actividad pesquera con seguridad. (Ver Figura 4)



Figura 3 Estrategia de abastecimiento

\section{Estrategia de cooperativa para el sector pesquero}

Esta estrategia cooperativa para el sector pesquero artesanal de los puertos de Chanduy, Santa Rosa y La caleta, dentro de las asociaciones llega a convertirse en un elemento fundamental en los recursos financieros para la gestión pesquera, consistiendo en un apoyo financiero considerable en las actividades de desarrollo 
económico, que gracias a ciertas actividades como es la pesca artesanal surge un ingreso sostenible para los socios y sus familias.

La estrategia representa a un proceso que puede traer buenos beneficios para la comunidad pesquera, como:

Obtener herramientas e insumos pesqueros de forma rápida y a precios accesibles.

\section{Materiales y métodos}

"Se efectúan, normalmente, cuando el objetivo es examinar un tema o problema de investigación poco estudiado, del cual se tiene muchas dudas o no se ha abordado antes" (Franco, 2009).

Según estos autores los alcances exploratorios se utilizan comúnmente cuando los casos de investigación no son estudiados de manera minuciosa, o se desconoce del tema, que particularmente generan dudas; cabe recalcar que a través de este alcance se podrá constatar las premisas que servirán como base para el desarrollo del estudio sobre el modelo de asociatividad que contribuyen o afectan a los pescadores artesanales de los puertos pesqueros de la provincia Santa Elena como son: Chanduy, La Caleta de La Libertad y Santa Rosa.

"Consiste, fundamentalmente, en caracterizar un fenómeno o situación concreta indicando sus rasgos más peculiares $\mathrm{o}$ diferenciadores" (Morales, Alcances: Descriptivo, Exploratorio y Explicativo, 2010).

Mediante la investigación descriptiva se establecen las características del fenómeno estudiado, delimitando cada uno de sus rasgos y analizando cada una de las variables de una manera implícita y de esta forma poder obtener una investigación con mayor veracidad.

El enfoque descriptivo está encaminado al descubrimiento de los escasos modelos de asociatividad que existe en los puertos pesqueros como son: Chanduy, La Caleta La Libertad, y Santa Rosa. 
"Usa como base en la medición numérica y el análisis estadístico, para establecer patrones de comportamiento y probar teorías" (Hernández, 2011).

A través del enfoque cuantitativo se obtiene como resultado información específica, con datos que se recopilan de forma numérica, lo que otorga mayor veracidad en la medición de variables, resultados, comprobación de hipótesis, y patrones de comportamiento.

Cabe mencionar que en un estudio cuantitativo a través de los datos obtenidos se podrá tomar las mejores decisiones con respecto a las asociaciones, como también proyectar soluciones que se plantean dentro de cada una de las problemáticas en la investigación de los tres puertos pesqueros.

Enfoque cualitativo utiliza la recolección de datos sin medición numérica para descubrir o afinar preguntas de investigación en el proceso de interpretación (Hernández Sampieri, Fernández, \& Baptista, METODOLOGÍA DE LA INVESTIGACIÓN, 2014).

En el enfoque cualitativo los resultados se obtienen sin la necesidad que los datos sean recopilados u obtenidos en medición numérica; en este modelo de investigación se generaliza la contextualización del estudio, dado que, busca descubrir y afinar temas de escaso conocimiento que se abordan dentro de los puertos pesqueros artesanales como son: Chanduy, La Libertad, y Santa Rosa.

El proceso de recolección de datos utilizados en la investigación residió en las fuentes primarias y secundarias, las mismas no tuvieron limitantes, debido al enfoque mixto.

"La entrevista es una técnica para recabar datos. Es un instrumento técnico que adopta la forma de un dialogo coloquial" (Laura Días Bravo, 2013). Técnica comúnmente utilizada por los investigadores dado que brinda un enfoque diferente del contexto a analizar. Es así que la entrevista es parte fundamental de un proceso de estudio, dado que se la realiza a expertos en el caso de la investigación realizada estas fueron direccionadas al presidente y miembros de las asociaciones, ya que ellos poseen conocimientos veraces acerca de 
las problemáticas a investigar, facilitando un análisis a fondo de las variables.

La observación consiste en el registro sistemático, válido y confiable del comportamiento o de la conducta manifiesta, la cual puede utilizarse en muy diversas circunstancias (Núñez, 2005).

Práctica que consiste en observar, recopilar y analizar los distintos contratiempos del entorno estudiado; es la forma de apreciar los hechos, de cómo se presentan, ya sea de una manera espontánea como también consignarlos por escrito. Consiste en ver sucesos y fenómenos que se quieren investigar, constituyendo positivamente al investigador obteniendo el mayor número de datos. Dentro del entorno se verificó que no existe un apropiado manejo de los conflictos, ya que, ellos mismos determinan las posibles soluciones de cada problemática, la división del trabajo, además de mantenerse en contacto durante la realización de la actividad pesquera. Sin embargo, los estatutos internos con los que cuentan las asociaciones permiten separar a los pescadores que incumplan o hagan caso omiso de las reglas.

"La encuesta es un procedimiento que permite explorar cuestiones que hacen a la subjetividad y al mismo tiempo obtener esa información de un número considerable de personas" (Grasso, 2006). Son series de preguntas que se realizan a un determinado número de personas, las cuales son elegidas al azar entrelazando criterios personales sobre el tema a analizar, la recaudación de la información ayuda a determinar cuál es la proporción de personas que pertenecen a una asociación pesquera y los beneficios que brindan cada una de ellas; igualmente los años de experiencia del pescador, factores que influyen en la pesca como inseguridad en alta mar y los y los escases de recursos.

\section{Resultados}

Los resultados del trabajo de investigación han permito realizar un diagnóstico de las poblaciones que han sido objeto de estudio, y su relación con la actividad de pesca artesanal, los mismo que son la base para determinar los procesos de socialización, para que los pescadores puedan coexistir en una asociación que les permita 
desarrollar sus actividades de forma organizada, bajo la dirección de lineamientos que les permitan cumplir con las normativas y regulaciones que la ley establece para este tipo de organizaciones sociales.

Estrategia de desarrollo para la asociatividad: Fortalecimiento de la actividad productiva.

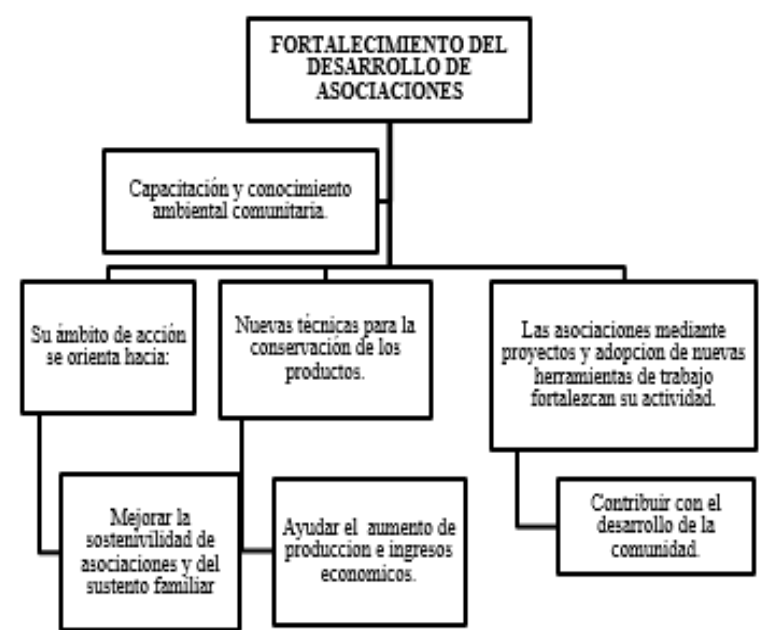

Figura 4 Fortalecimiento del desarrollo de asociaciones

La preparación permanente mediante capacitaciones que garanticen la sostenibilidad de la actividad de pesca artesanal, considerando un mejor cuidado del ambiente y tratado de las especies marinas, teniendo a su vez una mejor comercialización de las mismas, tanto así que les permita obtener múltiples beneficios por parte de las instituciones de gobierno, con la finalidad de que puedan aportar en el desarrollo comunitario como también lograr el fortalecimiento del sector pesquero.

Con la implementación de un modelo de gestión que permita aplicar políticas de asociatividad se normalizan las actividades, y se regulan sus miembros, orientados a un fin común, que busque mejores condiciones para sus integrantes, que exista apoyo gubernamental, y aprovechar las ventajas de las asociaciones de grupos sociales dentro de estas importantes regiones del País. 


\section{Discusión}

Los modelos de asociatividad organización de sectores de la sociedad, muchas veces son confundidas con grupos sindicales que buscan protagonismos y reclamos de derechos donde no existen o están fuera de la ley, este paradigma se ha venido manteniendo en la República del Ecuador en las últimas décadas, e incluso se han llegado a prohibir manifestaciones de grupos sociales que alteren el orden público, sin embargo las políticas cambiantes de los últimos 10 años, están generando nuevas oportunidades a la sociedad, para que se organicen y puedan velar bajo normativas establecidas, por el cumplimiento de sus derechos y que se garantice la libre pronunciación de la exigencia de sus derechos de forma individual y colectiva, por lo que surgen las siguientes interrogantes:

¿Están listas las agrupaciones sociales para enfrentar las políticas cambiantes en materia de crecimiento socio económico en el País?.

¿Se encuentran preparados los líderes sindicales, gremiales y asociativos, para llevar a sus organizaciones a la adopción de nuevos modelos de gestión organizacional?.

\section{Conclusiones}

En los puertos de Chanduy, Santa Rosa y La caleta se pudo constatar que existe una desorganización e inseguridad en la actividad pesquera, razón por la cual es importante implementar un modelo de asociatividad en estos sectores, en el transcurso del tiempo se ha ido desintegrando por la administración de turno perjudicando el desarrollo óptimo de las asociaciones o cooperativas.

Se determinó el FODA de los tres puertos, dando como resultado que el conocimiento de los pescadores es escaso para ejercer la actividad comercial provocando bajos costos de la materia prima ya que carecen de maquinarias para la conservación, debido a esto resulta una competencia desleal por parte de los pescadores al momento de realizar la comercialización.

Se pudo concluir que la implementación de las estrategias es importante para las asociaciones ya que esto permite asegurar la sostenibilidad de los recursos manteniendo activa la pesca artesanal 
de una manera eficiente y eficaz permitiendo múltiples beneficios que aportan al desarrollo comunitario.

Se elaboró un organigrama detallado para la implementación dentro las asociaciones, acerca de sus funciones, cadena de mando y el grado en que se toma las decisiones en cada nivel jerárquico.

La implementación de un modelo de asociatividad ayudara a un mejor desarrollo económico en la actividad pesquera, además de mejorar la administración en la asociatividad teniendo mayor beneficio los pescadores y sus familias.

Por medio del modelo de asociatividad se implementará un plan estratégico que permite asegurar los recursos económicos como también los recursos marítimos que brindan los tres puertos de Chanduy, Santa Rosa y La caleta obteniendo beneficios para una mejor rentabilidad de una manera eficiente y eficaz en la pesca artesanal.

Seguir un plan de acción para asegurar que la visión de la asociación se concrete, aplicando estrategias para el alcance de los objetivos en el arte pesquero trabajando de una forma organizada y responsable para el bien común de los pescadores.

La asociación debe mantenerse estable, para lograr este objetivo la administración debe conocer la importancia del análisis FODA, y de esa manera innovar, además de estar actualizados respecto a la estructura de la asociación o cooperativa.

\section{Referencias}

Baigún. (2013). La pesca artesanal y el desarrollo sostenible de la parroquia. Obtenido de la pesca artesanal y el desarrollo sostenible de la parroquia: http://repositorio.upse.edu.ec/bitstream/46000/4008/1/UPSE-TOD2016-0009.pdf

Banco Central del Ecuador. (2016). Obtenido de Banco Central del Ecuador: https://www.bce.fin.ec/index.php/boletines-de-prensaarchivo/item/975-producto-interno-bruto-2

Barreto Tajada, J. A., Cortes Millan , G. A., Bonilla Londoño, N. L., \& Rojas Beltran, F. M. (s.f.). Asociatividad: estrategia para el desarrollo nacional: Propuesta de politica ... 
Biblioteca Univesidad Tecnologica de El Salvador. (2003).

Chanduy. (2018). Obtenido de http://chanduy.gob.ec/index.php/ctmenu-item-31/ct-menu-item-47

Elizondo, A. (2002). Metodología de la Invetigación Contable. México: International Thomson Editores, S.A.

Elizondo, A. (2015). Metodología de la Invetigación Contable. México: International Thomson Editores, S.A.

Franco, J. (2009). Asociatividad empresarial y apropiación de la cadena productiva como factores que impulsan la competitividad.

Gabriela, L. (2008). "La economía informal”. CEPAL. Obtenido de "La economía informal”. CEPAL.

Grasso, L. (2006). Encuestas, Elemtos para su diseño y análisis . Editorial Brujas .

Hernández Sampieri, R., Fernández, C., \& Baptista, P. (2006). METODOLOGÍA DE LA INVESTIGACIÓN. México: McGraw-Hill.

Hernández Sampieri, R., Fernández, C., \& Baptista, P. (2014). METODOLOGÍA DE LA INVESTIGACIÓN. México: McGraw-Hill.

Hernández, L. D. (25 de Mayo de 2011). Asociatividad empresarial y apropiación de la cadena productiva como factores que impulsan la competitividad de las micro, pequeñas y medianas empresas: tres estudios de casos. Obtenido de Asociatividad empresarial y apropiación de la cadena productiva como factores que impulsan la competitividad de las micro, pequeñas y medianas empresas: tres estudios de casos: http://www.pa.gob.mx/publica/rev_47/an\%C3\%A1lisis/asociativid ad_empresarial.pdf

HINESTRO, M. P. (2005). PROCESOS DE SOCIATIVIDAD EMPRESARIAL APROXIMACIONES CONCEPTUALES E IMPACTO ECONOMICOS. (pág. 16). CEEP.

Jiménez Paneque, R. (1998). TIPOS DE INVESTIGACIONES SEGÚN ESTADO DEL CONOCIMIENTO Y ALCANCE. METODOLOGÍA DE LA INVESTIGACIÓN, 12. 
Laura Días - Bravo, U. T.-G.-H.-R. (2013). La entrevista, recurso flexible y dinámico . SciELO.

LIENDO, M. G., \& MARTINEZ, A. M. (NOVIEMBRE de 2001). ASOCIATIVIDAD UNA ALTERNATIVA PARA EL DESARROLLO $Y$ CRECIMIENTO DE LAS PYMES. Obtenido de ASOCIATIVIDAD UNA ALTERNATIVA PARA EL DESARROLLO Y CRECIMIENTO DE LAS PYMES: https://www.fcecon.unr.edu.ar/web/sites/default/files/u16/Decimoc uartas/Liendo,\%20Martinez_asociatividad.pdf

López , C., López, L., Pineda, J., \& Venegas, S. (2010). Vías y escenarios de la transformación laboral. Aproximaciones teóricas y nuevos problemas, Bogotá.

Lozano Monroy, F. D. (s.f.). LA ASOCIATIVIDAD COMO MODELO DE GESTIÓN PARA PROMOVER LAS EXPORTACIONES EN LAS PEQUEÑAS Y MEDIANAS EMPRESAS EN COLOMBIA. SCIELO.

MARCO HERRERA, R. C. (MAYO de 2015). PUERTOS, CALETAS Y ASENTAMIENTOS PERSQUEROS ARTESANALES DEL ECUADOR. Obtenido de PUERTOS, CALETAS Y ASENTAMIENTOS PERSQUEROS ARTESANALES DEL ECUADOR: $\quad$ http://www.institutopesca.gob.ec/wpcontent/uploads/2015/05/INP_BE_A4_N1.pdf

Morales, F. (Mayo de 2010). Alcances: Descriptivo, Exploratorio y Explicativo. Pensamiento Imaginativo.

Morales, F. (Mayo de 2013). Alcances: Descriptivo, Exploratorio y Explicativo. Pensamiento Imaginativo.

Perez Villa, P. E., \& Munera Vasquez, F. N. (s.f.). Reflexiones para implementar un sistema de gestión de calidad (ISO 9001: 2000) en cooperativas y empresas de economía solidaria. Universidad Cooperativa de Colombia.

Ramon Valdez, L. (2000). Comunidades Productivas: Asociatividad y Producción en el Territorio.

Razo, C. M. (2011). Como elaborar y asesorar una investigación de tesis. Mexico: Pearson. 
Risea, S. (2013). Plan de asociatividad para la asociación de pequeños comerciantes de artesania. Unibiblos.

Salazar, H. Z. (2007). Asociatividad, Redes Sociales y Desarrollo Local. Fundacion Universitaria Luis Amigo de Colombia.

Sampieri, R. H. (23 de Marzo de 2011). Metodolgía de la Investigación. México: Capítulo 5 Sampieri. Obtenido de Metodología de la Investigación: https://sites.google.com/site/metodologiadelainvestigacionb7/capitu lo-5-sampieri

Sampieri, R. H. (23 de Marzo de 2013). Metodolgía de la Investigación. México: Capítulo 5 Sampieri. Obtenido de Metodología de la Investigación: https://sites.google.com/site/metodologiadelainvestigacionb7/capitu lo-5-sampieri

TELEGRAFO, E. (23 de ENERO de 2108). Los sectores pesquero y acuícola aportaron en 2013 el 0,04\% al PIB, que se situó en 4,5\%. Obtenido de Los sectores pesquero y acuícola aportaron en 2013 el $0,04 \%$ al PIB, que se situó en $4,5 \%$ : http://www.eltelegrafo.com.ec/noticias/economia/8/la-pescaartesanal-genera-100-millones-en-exportaciones-al-ano

Torres, C. B. (2010). Metodología de la Investigación. Colombia: Pearson.

Torres, C. B. (2013). Metodología de la Investigación. Colombia: Pearson.

Torres, C. B. (2013). Metodología de la investigación. México: Pearson Educación. 